\title{
COMPOSIÇÃO FLORÍSTICA DE UM TRECHO DE CERRADÃO E CERRADO SENSU STRICTO E SUA RELAÇÃO COM O SOLO NA FLORESTA NACIONAL (FLONA) DE PARAOPEBA, MG, BRASIL ${ }^{1}$
}

\author{
Érica Pereira de Campos², Temilze Gomes Duarte², Andreza Viana Neri², Alexandre Francisco da Silva ${ }^{4}$,
} João Augusto Alves Meira-Neto ${ }^{4}$ Gilmar Edilberto Valente ${ }^{5}$

\begin{abstract}
RESUMO - O presente trabalho foi realizado na FLONA de Paraopeba, MG, e teve como objetivo o levantamento florístico das fanerófitas, ao longo de um gradiente de cerradão e cerrado sensu stricto, em uma área de 2.600 $\mathrm{m}^{2}$. Foram encontradas 91 espécies, pertencentes a 71 gêneros de 41 famílias. As famílias mais representativas foram Leguminosae, Myrtaceae, Malpighiaceae, Vochysiaceae, Rubiaceae e Melastomataceae. Os gêneros Miconia, Myrcia, Erythroxylum e Qualea foram os mais ricos. Magonia pubescens destacou-se em número de indivíduos. A similaridade florística mostrou a separação das parcelas em dois grupos, em que o primeiro apresentou um nível de similaridade de cerca de $45 \%$, e o segundo foi dividido em dois grandes subgrupos, sendo que o primeiro mostrou nível de similaridade de cerca de $38 \%$, enquanto as demais parcelas não formaram grupos definidos. A ordenação das espécies pela análise de correspondência canônica sugeriu que Magonia pubescens, Bauhina holophylla e Terminalia brasiliensis tenderam a ser mais abundantes nas áreas com valores mais altos de $\mathrm{pH}$, $\mathrm{Ca}, \mathrm{Mg}$ e H+Al. A variação não explicada das demais espécies pode estar associada a outras variáveis não analisadas, além de um complexo conjunto de fatores que estão envolvidos na determinação da composição da vegetação.
\end{abstract}

Palavras-chave: Cerrado, levantamento florístico e relação solo-vegetação.

\section{FLORISTIC COMPOSITION OF A STRETCH OF “CERRADO” SENSU STRICTO AND “CERRADÃO” AND ITS RELATION WITH SOIL IN THE 'FLORESTA NACIONAL' (FLONA) OF PARAOPEBA, MG}

\begin{abstract}
The objective of this work was to carry out the floristic survey of phanerophytes along a gradient of "cerrado sensu stricto " and "cerradão" in an area of 2,600m ${ }^{2}$ in the FLONA (Floesta Nacional-National Forest) of Paraopeba, MG, Brazil. A total of 91 species pertaining to 71 genera and 41 families were found. The most representative families were Leguminosae, Myrtaceae, Malpighiaceae, Vochysiaceae, Rubiaceae and Melastomataceae. Miconia, Myrcia, Erythroxylum and Qualea were the richest genera. Magonia pubescens was outstanding in number of individuals. The floristic similarity showed the separation of the plots in two groups, the first one showed a similarity level around $45 \%$, and the second group was divided into two large groups, the first one showed a similarity level around $38 \%$ and the remaining plots did not form definite groups. The species ordination by canonical correspondence analysis suggests that Magonia pubescens, Bauhinia holophylla and Terminalia brasiliensis tended to be most abundant species in areas with higher values of $p H, M g, H+A l$. The non-explained variation for the other species could be associated to other non-analyzed variables as well as to a complex set of factors that are involved in the vegetation composition determination.
\end{abstract}

Keywords: "Cerrado", floristic survey and soil-vegetation relation.

\footnotetext{
${ }^{1}$ Recebido em 15.12.2004 e aceito para publicação em 05.04.2006.

${ }^{2}$ Programa de Pós-Graduação em Botânica da UFV. E-mail:<camposep@ig.com.br>.

${ }^{4}$ Departamento de Biologia Vegetal da UFV. 36570-000 Viçosa-MG. E-mail:<afsilva@ufv.br>.

${ }^{5}$ Técnico do Departamento de Biologia Vegetal da UFV.
} 


\section{INTRODUÇÃO}

Segundo Coutinho (1978), do ponto de vista fisionômico, os cerrados apresentam dois extremos: o cerradão, fitofisionomia na qual predomina o componente arbóreo-arbustivo, e o campo limpo, onde há predomínio do componente herbáceosubarbustivo. As demais fitofisionomias encontradas - campo sujo, campo cerrado, cerrado (sentido restrito) - podem ser consideradas ecótonos entre o cerradão e o campo limpo.

Lopes (1984) citou que a distribuição da flora, além de ser condicionada pelos fatores básicos como clima, características químicas e físicas do solo, disponibilidade de água e nutrientes, é fortemente influenciada pela latitude, freqüência de queimadas, profundidade do lençol freático, pastejo e inúmeros fatores antrópicos.

Para Martins (1990), o levantamento florístico é um dos estudos iniciais para o conhecimento da flora de determinada área e implica produção de uma lista de espécies ali instaladas, que poderão contribuir para o estudo dos demais atributos da comunidade.

Vários estudos da flora lenhosa já foram realizados em áreas de Cerrado (RIZZINI, 1971; RIBEIRO et al.,1985; MANTOVANI e MARTINS, 1993; COSTA e ARAÚJO, 2001; WEISER e GODOY, 2001). Esses trabalhos evidenciaram a importância do bioma Cerrado, as suas fitofisionomias típicas e a elevada diversidade florística, sobretudo da flora arbustivo-arbórea, muito significativa e variada em relação aos outros estratos.

Os estratos arbustivo e arbóreo são classificados por Raunkier apud Braun-Blanquet (1979), na forma de vida fanerófita. O levantamento das fanerófitas dentro de um gradiente que se estende do cerradão ao cerrado s.s. permite conhecer a composição florística de cada fitofisionomia, bem como a sua variação ao longo do gradiente.

O conhecimento da composição florística em área demarcada abre perspectivas para o desenvolvimento de pesquisas relacionadas à fitossociologia, à fenologia e à dinâmica das populações ali instaladas. Do mesmo modo, o amplo conhecimento da flora do bioma Cerrado é um importante subsídio para o planejamento e implementação de áreas representativas desse bioma, que devem ser priorizadas para conservação e manejo racional (FELFILI et al., 1993 e MENDONÇA et al., 1998).
Dessa forma, o presente trabalho teve como objetivos determinar a composição florística das fanerófitas e correlacioná-la com algumas variáveis de solo em um trecho de cerradão e cerrado $s$. $s$. da Floresta Nacional de Paraopeba, MG.

\section{MATERIAL E MÉTODOS}

\section{Área de Estudo}

A Floresta Nacional de Paraopeba (FLONA), Paraopeba, MG, Brasil, está situada a 19²0'S e 4420'W, em altitude que varia dos 734 aos $750 \mathrm{~m}$, apresentando uma área total de aproximadamente 200 ha, divididos em 150 ha de remanescentes de cerrado e 50 ha destinados à implantação de vários experimentos.

O clima é do tipo subtropical úmido, com verão chuvoso e estação seca que varia de abril a setembro (THORNTHWAITE MATHER, 1957, citados por SILVA JÚNIOR, 1984).

Os solos da FLONA foram classificados por Thibau et al. (1975) como Latossolo Vermelho-Escuro, Latossolo Vermelho-Amarelo e Latossolo Amarelo.

Segundo Silva Júnior (1984), houve desmatamento total dos 200 ha em 1952 e há registro de fogo nos anos de 1960 e 1963.

\section{Composição Florística}

Com o objetivo de verificar a variação da composição florística ao longo das fitofisionomias de cerradão e cerrado s.s. adjacentes, foram instalados dois transectos paralelos de 10 x 130 m cada, subdivididos em 13 parcelas de $10 \times 10 \mathrm{~m}$, ao longo do gradiente vegetacional, totalizando $2.600 \mathrm{~m}^{2}$ (Figura 1). Foram amostrados todos os indivíduos arbustivos e arbóreos com altura superior ou igual a 0,25 m, denominados fanerófitas, segundo Raunkier apud Braun-Blanquet (1979).

O sistema de classificação adotado foi o de Cronquist (1988), exceto na família Leguminosae, para a qual foram mantidas as três subfamílias.

\section{Análise de Similaridade}

Para comparar as parcelas quanto à similaridade florística, foi construída uma matriz de presença e ausência das espécies nas parcelas. A partir dessa matriz foi calculada a similaridade florística através do índice deSorensen(MÜELLER-DOMBOIS eELLENBERG, 1974), e a interpretação dos dados se deu pelo método média 
de grupo (UPGMA) (SNEATH e SOKAL, 1973). As análises foram feitas utilizando o programa FITOPAC 1 (SHEPHERD, 1996).

\section{Análises de Solo}

Para a análise química do solo foram coletadas três amostras simples para formar uma amostra composta para cada parcela da faixa 2 . As amostras foram feitas na profundidade de $0-20 \mathrm{~cm}$, onde foram analisados $\mathrm{pH}, \mathrm{P}, \mathrm{K}, \mathrm{Ca}, \mathrm{Mg}, \mathrm{Al}$ e H+Al. As análises químicas foram realizadas no Laboratório de Solos da Universidade Federal de Viçosa, em Viçosa, MG.

\section{Ordenação de dados de solo e vegetação}

Para a análise das interações entre a abundância de espécies e a variação do solo, foi utilizada a análise de Correspondência Canônica (CCA), empregandose o programa PC-ORD (McCUNE e MEFFORD, 1997). O teste de permutação de Monte Carlo foi utilizado para avaliar o nível de significância dos eixos de ordenação.

Foram consideradas apenas as espécies com seis ou mais indivíduos no levantamento, totalizando 20 espécies arbóreas. As variáveis ambientais utilizadas foram: $\mathrm{pH}, \mathrm{Ca}, \mathrm{Mg}$ e $\mathrm{H}+\mathrm{Al}$.

\section{RESULTADOS E DISCUSSÃO}

Foram encontradas 91 espécies pertencentes a 71 gêneros de 41 famílias (Quadro 1). As famílias mais representativas floristicamente foram Leguminosae (14), Myrtaceae (6), Malpighiaceae (5), Vochysiaceae (5), Rubiaceae (5) e Melastomataceae (4), representando $41,76 \%$ das espécies amostradas; o restante das espécies $(58,24 \%)$ estava distribuído entre 35 famílias, demonstrando a alta riqueza desses taxa.

A representatividade dessas famílias também foi verificada por Goodland (1969) no Triângulo Mineiro, Felfili et al. (1993) na Chapada Pratinha (DF) e Weiser e Godoy (2001) na ARIE Cerrado Pé-de-Gigante (SP). Segundo Silva (2002), Leguminosae tem sido a família mais diversificada na maioria dos levantamentos realizados no cerrado.

Nesse levantamento foi verificado elevado número de gêneros, sendo Miconia e Myrcia, com quatro espécies cada; e Erythroxylum e Qualea com três, os mais ricos. Aspidosperma, Bauhinia, Byrsonima, Eugenia, Guapira, Kielmeyera, Ouratea, Styrax, Tabebuia, Terminalia e Vernonia apresentaram duas espécies cada um e o restante dos gêneros (55), uma espécie.

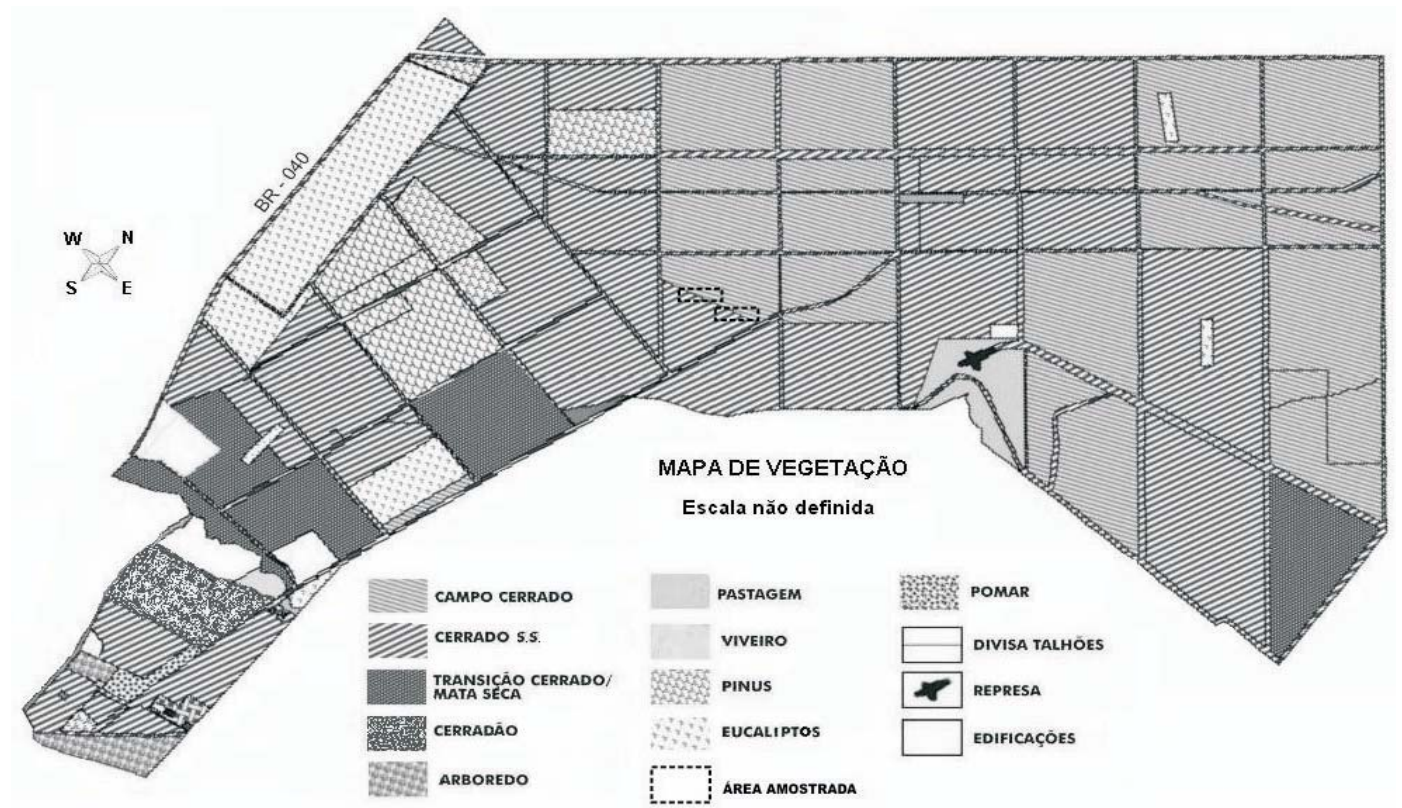

Figura 1 - Croqui da Floresta Nacional de Paraopeba, MG, com a localização das parcelas amostradas. Figure 1-- Outline of the Paraopeba Nacional Forest, MG, with the location of the sampled plots. 
Quadro 1 - Famílias e espécies encontradas em uma área de $2.600 \mathrm{~m}^{2}$, em um gradiente de cerradão e cerrado s.s. na FLONA de Paraopeba, MG, Brasil

Table 1 - Families and species found in an 2,600 $\mathrm{m}^{2}$ area in a gradient of "cerrado s.s." and "cerradão", in the FLONA of Paraopeba, $M G$

Anacardiaceae

Astronium fraxinifolium Schott.

Tapirira guianensis Aubl.

Annonaceae

Annona crassiflora Mart.

Xylopia aromatica (Lam.)Mart.

Apocynaceae

Aspidosperma subincanum Mart.

Aspidosperma tomentosum (A DC.) Mart.

Araliaceae

Schefflera macrocarpa (Seem.) DC. Frondim

Asteraceae

Piptocarpha rotundifolia (Less.) Baker

Vernonia rubriranea Mart.

Vernonia sp.

Bignoniaceae

Tabebuia aurea (Manso) Benth. \& Hook. F. ex S. Moore

T. caraiba (Mart.) Bureau

Zeyheria digitalis (Vell.) Hoehne.

Bombacaceae

Pseudobombax grandiflorum A. Rob

Burseraceae

Protium heptaphyllum March.

Caryocaraceae

Caryocar brasiliense Camb.

Celastraceae

Austroplenckia populnea (Reiss.) Lund.

Clusiaceae

Kielmeyera coriacea (Spreng.) Mart.

K. rublifora A. St. Hil.

Combretaceae

Terminalia argentea Mart. \& Zucc.

T. brasiliensis Eichler

Connaraceae

Connarus suberosus Planch.

Dilleniaceae

Curatella americana $\mathrm{L}$.

Davilla rugosa Poiret

Ebenacea

Diospyros hispida A. DC.

Erythroxilaceae

Erythroxylum daphinites Mart.

E. suberosum St. Hil.

E. tortuosum Mart.

Euphorbiaceae

Manihot tripartita Müll. Arg.

Pera glabrata (Schott.) Baill.

Flacourtiaceae

Casearia sylvestris Sw

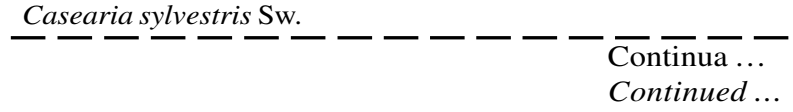

Quadro 1 - Cont.

Table 1 -Cont.

\section{Lamiacea}

Hyptis cana Pohl ex Benth.

Lauraceae

Ocotea corymbosa (Meisn.) Mez

Leguminosae - Caesalpinioideae

Bauhinia holophylla Steud

Bauhinia forficata Link

Copaifera langsdorffii Desf.

Hymenaea stigonocarpa Mart. ex Hayne

Sclerolobium aureum (Tul.) Benth.

Senna rugosa Irwin \& Barneby

Leguminosae - Mimosoideae

Dimorphandra mollis Benth.

Enterolobium gumiferum J. Macbr.

Plathymenia reticulata Benth.

Stryphnodendron adstringens (Mart.) Cov.

Leguminosae - Papilionoideae

Acosmium dasycarpum (Vog.) Yaovl.

Bowdichia virgilioides H. B. \& K.

Machaerium opacum Vog.

Platypodium elegans Vog.

Malpighiaceae

Banisteriopsis gardneriana (A. Juss.) W. R. Anderson \& B. Gates Byrsonima coccolobifolia H. B. \& K.

B. verbascifolia (L.) Rich.ex A. L. Juss.

Heteropteris sp.

Pterandra piroidea A. Juss.

Malvaceae

Pavonia malacophylla (Link \& Otto) Garcke

Melastomataceae

Miconia albicans (Sw.) Triana

M. fallax DC.

M. rubiginosa (Bonpl.) DC.

Miconia sp.

Meliaceae

Cabralea cangerana (Vell.) Mart.

Monimiaceae

Siparuna guianensis Aubl.

Moraceae

Brosimum gaudichaudii Tréc.

Myrsinaceae

Cybianthus detergens Mart.

Myrtaceae

Campamonesia pubescens (DC.) O. Berg.

Eugenia bimarginta DC.

Eugenia dysenterica DC.

Myrcia formosiana DC.

M. lingua Berg.

M. rostrata $\mathrm{DC}$.

M. tomentosa (Aubl.) DC. 
Quadro 1- Cont.

Table 1 - Cont.

\section{Nyctaginaceae}

Guapira ferruginea (Klotzsch ex Choisy Lundell)

G. noxia (Netto.) Luna.

Neea theifera Oerst.

Ochnaceae

Ouratea castaneaefolia Engl.

O. spectabilis (Mart.) Engl.

Opiliaceae

Agonandra brasiliensis Beth. \& Hook. F.

Proteaceae

Roupala montana Aubl.

Rubiaceae

Alibertia edulis (L.Rich.) A.Rich.

Palicourea rigida $\mathrm{H}$. B. K.

Rudgea viburnoides (Cham.) Benth.

Sabicea sp.

Tocoyena formosa (Cham. \& Schlecht.) K. Schum

Sapindaceae

Magonia pubescens St. Hil.

Solanaceae

Cestrum sp.

Styracaceae

Styrax camporum Pohl

S. ferrugeneus Nees \& Mart.

Symplocaceae

Symplocos nitens Benth.

Verbenaceae

Aegiphila lhotskyana Cham.

Vochysiaceae

Callisthene major Mart.

Qualea grandiflora Mart.

Q. multiflora Mart.

Q. parviflora Mart.

Vochysia rufa Mart.

Qualea grandiflora distribuiu-se entre as duas fitofisionomias, corroborando as observações de Ratter e Dargie (1992) e Ratter et al. (1996), que, ao analisarem trabalhos de levantamentos florísticos em áreas de cerrado, observaram que essa espécie foi a mais amplamente distribuída, estando tanto em áreas de cerrado sensu stricto, cerradão e até mesmo em campo cerrado (embora com densidades mais baixas).

Magonia pubescens, espécie característica do Cerrado, ocorre principalmente em cerradões com melhores condições pedológicas (RATTER et al., 1977), foi o destaque em número de indivíduos. Segundo Silva Júnior e Silva (1988), em levantamento fitossociológico realizado na FLONA de Paraopeba, MG, a densidade absoluta dessa espécie foi de 56 indivíduos por hectare.
De acordo com estudo fitossociológico realizado por Balduino (2005), no mesmo local, Magonia pubescens teve ocorrência restrita na amostragem, situando-se no grupo das espécies que apresentaram os menores valores de freqüência relativa $(0,28)$. Quanto à dominância e à densidade relativas, essa espécie chegou a valores maiores, melhorando a sua colocação em termos de comparação com o VI das demais espécies.

Segundo Costa e Araújo (2001), Miconia albicans, Myrcia rostrata e Xylopia aromatica são espécies que ocorrem com elevada densidade em cerrados alterados do Município de Uberlândia, o que pode indicar que o cerradão e o cerrado s.s. da Reserva do Panga - local onde foi realizado o estudo - possam estar se recuperando do impacto de eventuais ações antrópicas que ocorreram no passado.

Situação semelhante pode estar ocorrendo na FLONA de Paraopeba devido, principalmente, à ação de "minhoqueiros", que revolvem as primeiras camadas do solo em busca dos minhocuçus (Oligochaeta), cortando, ao mesmo tempo, as plântulas e indivíduos jovens, alterando a dinâmica da comunidade.

O dendrograma de similaridade florística (Figura 2) mostrou a separação das parcelas em dois grupos. O primeiro apresentou um nível de similaridade de cerca de $45 \%$, sendo formado pelas parcelas $9,12,15$ e 24, com as seguintes espécies comuns: Terminalia argentea, Qualea parviflora, Byrsonima verbascifolia, Erythroxylum daphinites, Erythroxylum suberosum e Magonia pubescens.

A separação desse grupo provavelmente se deu pela ocorrência de Myrcia formosiana, Stryphnodendron adstringens, Vochysia rufa e Davila rugosa.

O segundo grupo, formado pelas demais parcelas, foi dividido em dois grandes subgrupos, em que o primeiro mostrou um nível de similaridade de cerca de $38 \%$, sendo constituído pelas parcelas $1,6,21,2,19,3,4,5,18$, 23, 10 e 22, apresentando as seguintes espécies comuns: Qualea parviflora, Xylopia aromarica, Siparuna guianensis e Magonia pubescens. Cabe ressaltar que as parcelas $(1,2,3,4,5,6)$ da faixa 1 localizadas no cerradão fizeram parte do mesmo subgrupo, constituindo o agrupamento mais evidente; as demais parcelas não formaram grupos bem definidos, estando distribuídas entre os dois subgrupos.

R. Árvore, Viçosa-MG, v.30, n.3, p.471-479, 2006 


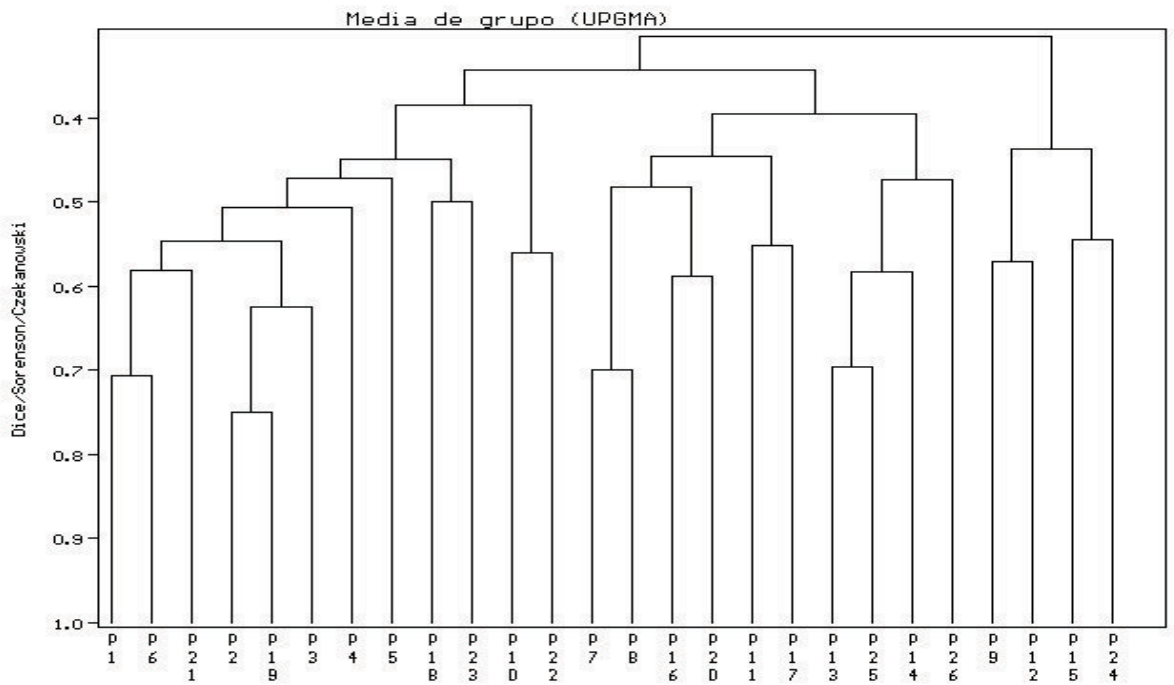

Figura 2 - Dendrograma de similaridade florística obtido pelo método média de grupo (UPGMA), com base no índice de Sorensen, para dados de presença e ausência das espécies nas 26 parcelas na FLONA de Paraopeba, MG, Brasil.

Figure 2 - Floristic similarity dendrogram obtained by the group average method (UPGMA), based on the Sorensen Index, for data of presence and absence of species in 26 plots in the FLONA of Paraopeba, MG, Brazil.

O solo das parcelas amostradas, classificado como Latossolo Vermelho-Amarelo, apresentou baixas condições de fertilidade, e os resultados das análises químicas (Quadro 2), segundo Tomé Júnior (1997), indicaram $\mathrm{pH}$ ácido e baixos teores de fósforo, cálcio, magnésio e teor médio de potássio.

$\mathrm{Na}$ análise de correspondência canônica, os autovalores para os eixos 1 e 3 foram, respectivamente, 0,304 e 0,140 , sendo que o eixo 1 explicou $24,7 \%$ da variância e o eixo 3, 11,4\%. Ter Braak (1988) considerou que baixas porcentagens de variação são esperadas em ordenações de dados ecológicos, tendo em vista a complexidade dos fatores envolvidos na determinação da composição florística e estrutural de formações vegetais.

As variáveis $\mathrm{Ca}, \mathrm{Mg}, \mathrm{pH}$ e $\mathrm{H}+\mathrm{Al}$ foram as que apresentaram as maiores variações ao longo das parcelas. Portanto, sendo utilizadas na CCA, essas variáveis apresentaram as maiores correlações com eixo 1, exceto para o pH, que teve maior correlação com o eixo 3 (Quadro 3).

Quadro 2 - Características químicas da camada superficial $(0-20 \mathrm{~cm})$ das parcelas do transecto 2 em um gradiente de cerradãocerrado $s$. s. na Floresta Nacional de Paraopeba, Paraopeba, MG, Brasil

Table 2 - Chemical characteristics of the superficial layer $(0-20 \mathrm{~cm})$ of the transect 2 in a gradient of "cerradão" and "cerrado s.s." in the FLONA of Paraopeba, MG, Brazil

\begin{tabular}{|c|c|c|c|c|c|c|c|c|c|c|c|c|}
\hline \multirow[t]{2}{*}{ Parcela } & \multirow{2}{*}{$\begin{array}{c}\mathrm{pH} \\
\mathrm{H}_{2} \mathrm{O}\end{array}$} & $\mathrm{P}$ & $\mathrm{K}$ & $\mathrm{Al}^{+3}$ & $\mathrm{Ca}^{+2}$ & $\mathrm{Mg}^{+2}$ & $\mathrm{H}+\mathrm{Al}$ & SB & $(\mathrm{t})$ & $(\mathrm{T})$ & $\mathrm{V}$ & $\mathrm{m}$ \\
\hline & & \multicolumn{2}{|c|}{$\mathrm{mg} / \mathrm{dm}^{3}$} & \multicolumn{7}{|c|}{$\mathrm{cmol}_{\mathrm{c}} / \mathrm{dm}^{3}$} & \multicolumn{2}{|c|}{$\%$} \\
\hline 1 & 4,28 & 0,6 & 76 & 3,50 & 0,39 & 0,35 & 8,4 & 0,93 & 4,43 & 9,33 & 10,0 & 79,0 \\
\hline 2 & 4,23 & 0,4 & 79 & 3,30 & 0,29 & 0,30 & 8,6 & 0,79 & 4,09 & 9,39 & 8,4 & 80,7 \\
\hline 3 & 4,24 & 0,3 & 80 & 3,40 & 0,16 & 0,20 & 8,3 & 0,56 & 3,96 & 8,86 & 6,3 & 85,9 \\
\hline 4 & 4,30 & 0,2 & 79 & 3,30 & 0,18 & 0,22 & 7,9 & 0,60 & 3,90 & 8,50 & 7,1 & 84,6 \\
\hline 5 & 4,43 & 0,1 & 64 & 3,30 & 0,09 & 0,17 & 6,9 & 0,42 & 3,72 & 7,32 & 5,7 & 88,7 \\
\hline 6 & 4,39 & 0,0 & 76 & 2,90 & 0,14 & 0,20 & 7,3 & 0,53 & $3,4 \overline{3}$ & 7,83 & 6,8 & 84,5 \\
\hline 7 & 4,49 & 0,7 & 60 & 3,10 & 0,18 & 0,23 & 6,8 & 0,56 & 3,66 & 7,36 & 7,6 & 84,7 \\
\hline 8 & 4,51 & 2,7 & 84 & 3,00 & 0,34 & 0,16 & 6,9 & 0,71 & 3,71 & 7,61 & 9,3 & 80,9 \\
\hline 9 & 4,45 & 0,8 & 90 & 2,90 & 0,15 & 0,15 & 6,9 & 0,53 & 3,43 & 7,43 & 7,1 & 84,5 \\
\hline 10 & 4,51 & 0,1 & 88 & 2,70 & 0,15 & 0,21 & 6,6 & 0,59 & 3,29 & 7,19 & 8,2 & 82,1 \\
\hline 11 & 4,54 & 0,1 & 106 & 2,70 & 0,26 & 0,27 & 6,6 & 0,80 & 3,50 & 7,40 & 10,8 & 77,1 \\
\hline 12 & 4,52 & 0,6 & 84 & 2,60 & 0,19 & 0,22 & 6,6 & 0,62 & 3,22 & 7,22 & 8,6 & 80,7 \\
\hline 13 & 4,61 & 0,5 & 80 & 2,90 & 0,09 & 0,13 & 5,9 & 0,42 & 3,32 & 6,32 & 6,6 & 87,3 \\
\hline
\end{tabular}

$\mathrm{SB}=$ soma de bases trocáveis; $\mathrm{t}=$ capacidade de troca catiônica efetiva; $\mathrm{T}=$ capacidade de troca catiônica a $\mathrm{pH} 7,0 ; \mathrm{V}=$ índice de saturação de bases; e $\mathrm{m}=$ índice de saturação de alumínio.

R. Árvore, Viçosa-MG, v.30, n.3, p.471-479, 2006 
Quadro 3 - Coeficientes de correlação entre as variáveis pedológicas e os dois eixos de ordenação da análise de correspondência canônica em um gradiente de cerradão e cerrado s.s. na FLONA de Paraopeba, MG, Brasil

Table 3 - Correlation coefficients among the soil variables and the two ordination axes of the Canonic Correspondence Analysis in a gradient of "cerradão." and "cerrado s.s." in the FLONA of Paraopeba, MG, Brazil

\begin{tabular}{ccc}
\hline Variáveis Pedológicas & Eixo 1 & Eixo 3 \\
\hline $\mathrm{PH}$ & 0,033 & 0,535 \\
$\mathrm{Ca}$ & 0,647 & 0,046 \\
$\mathrm{Mg}$ & 0,832 & 0,103 \\
$\mathrm{H}+\mathrm{Al}$ & 0,605 & $-0,138$ \\
\hline
\end{tabular}

A ordenação das espécies pela CCA indicou que Magonia pubescens, Bauhina holophylla, Aspidosperma tomentosum e Terminalia brasiliensis tenderam a ser mais abundantes nas parcelas com valores mais altos de $\mathrm{pH}, \mathrm{Ca}, \mathrm{Mg}$ e $\mathrm{H}+\mathrm{Al}$ (Figura 3). Os resultados encontrados para Magonia pubescens em relação ao Mg corroboraram os encontrados por Balduino (2005) na FLONA de Paraopeba. Quanto ao Ca, os resultados encontrados estão de acordo com os de Ratter et al. (1978) e Haridasan (1982, 1988). Silva Junior e Silva (1988) comentaram que, em estudos de relação plantasolo, Magonia pubescens foi considerada exigente quanto à fertilidade do solo, principalmente em relação ao $\mathrm{K}, \mathrm{Ca}$ e $\mathrm{Mg}$, sendo, portanto, indicadora de solo de maior fertilidade.

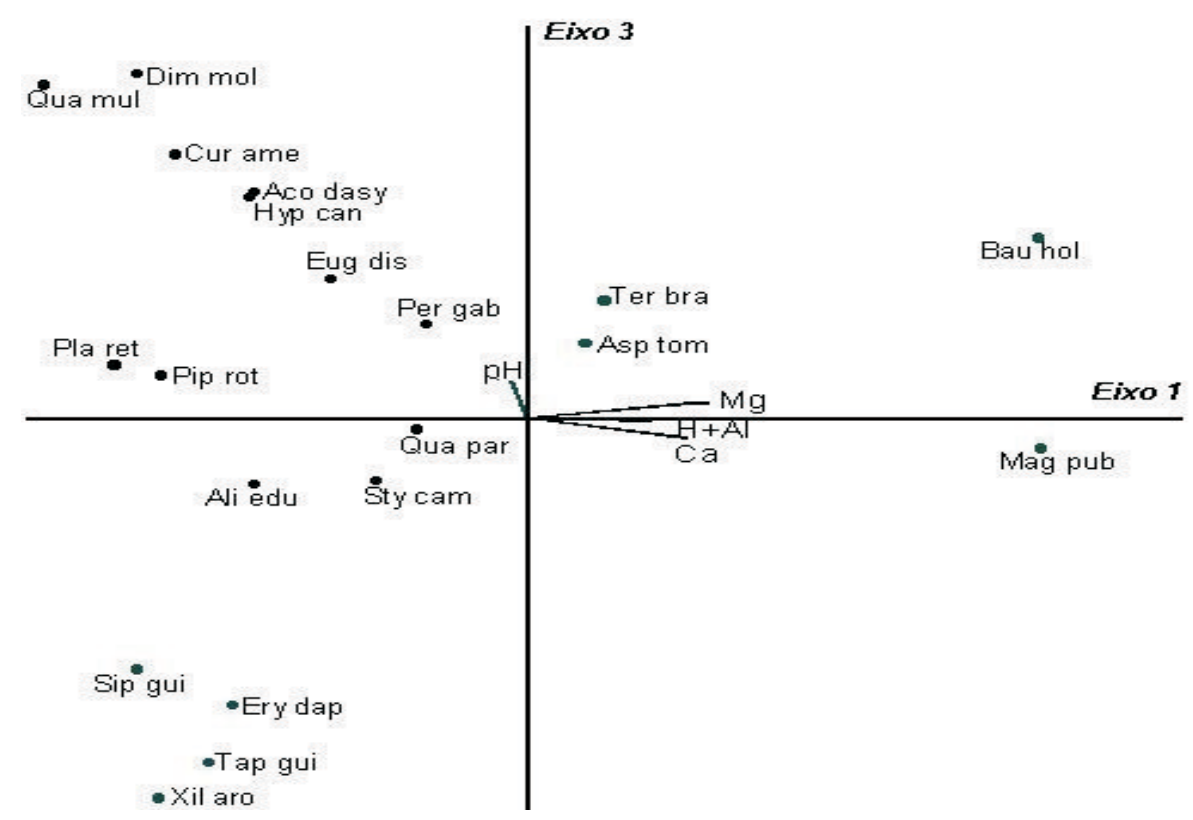

Figura 3 - Diagrama de ordenação produzido pela análise de correspondência canônica entre as espécies e as variáveis pedológicas dos eixos 1 e 3 em um trecho cerradão e cerrado s.s. na FLONA de Paraopeba, MG, Brasil. As variáveis edáficas estão representadas por vetores e as espécies, por seus nomes abreviados. Em que: Aco dasy =Acosmium dasycarpum, Ali edu =Alibertia edulis, Asp tom $=$ Aspidosperma tomentosum, Bau hol $=$ Bauhinia holophylla, Cur ame $=$ Curatella americana, Dim mol $=$ Dimorphandra mollis, Ery dap=Erythroxylum daphinites, Eug dis = Eugenia dysenterica, Hyp can = Hyptis cana, Mag pub=Magonia pubescens, Per gab = Pera glabrata, Pip rot $=$ Piptocarpha rotundifolia, Plat ret $=$ Plathymenia reticulata, Qua mul = Qualea multiflora, Qua par $=$ Qualea parviflora, Sip gui $=$ Siparuna guianensis, Sty cam $=$ Styrax camporum, Tap gui $=$ Tapirira guianensis, Ter bra $=$ Terminalia brasiliensis e Xil aro $=$ Xylopia aromatica .

Figure 3- Ordination diagram obtained by the Canonic Correspondence Analysis among the species and soil variables for axes 1 and 3 in a stretch of "cerrado s.s" and cerradão in the FLONA of Paraopeba, MG, Brazil. The soil variables are represented by vectors and the species are represented by their abbreviated names, as follows: Aco dasy =Acosmium dasycarpum, Ali edu $=$ Alibertia edulis, Asp tom = Aspidosperma tomentosum, $\mathrm{Bau}$ hol = Bauhinia holophylla, Cur ame $=$ Curatella americana, Dim mol = Dimorphandra mollis, Ery dap = Erythroxylum daphinites, Eug dis = Eugenia dysenterica, Hyp can = Hyptis cana, Mag pub = Magonia pubescens, Per gab $=$ Pera glabrata, Pip rot $=$ Piptocarpha rotundifolia, Plat ret $=$ Plathymenia reticulata, Qua mul = Qualea multiflora, Qua par $=$ Qualea parviflora, Sip gui $=$ Siparuna guianensis, Sty cam $=$ Styrax camporum, Tap gui $=$ Tapirira guianensis, Ter bra $=$ Terminalia brasiliensis, Xil aro $=$ Xylopia aromatica . 
Balduino et al. (2005) encontrou Magonia pubescens, Diospyros hispida, Aspidosperma macrocarpon e Siparuna guianensis, associando-se aos maiores valores de magnésio. Essas espécies formaram um bloco isolado das demais, evidenciando a forte influência das condições edáficas (principalmente $\mathrm{Mg}$ ) no sítio de ocorrência.

A variação não explicada para as demais espécies pode estar associada a outras variáveis não-analisadas, como a acentuada interferência dos "minhoqueiros", que revolvem as camadas mais superficiais do solo, alterando a estrutura, além de um complexo conjunto de fatores que estão envolvidos na determinação da composição da vegetação.

A FLONA de Paraopeba é uma das poucas unidades de conservação da região, daí a importância dos dados apresentados, fornecendo subsídios para estudos futuros, visando à preservação de seus recursos genéticos e da preservação de uma das áreas representativas de cerrado no Estado de Minas Gerais.

\section{REFERÊNCIAS BIBLIOGRÁFICAS}

BALDUINO, A. P. C. et al. Fitossociologia e análise comparativa da composição florística do cerrado da flora de Paraopeba-MG. Revista Árvore, v. 29, n. 1, p. 25-34, 2005.

BRAUN-BLANQUET, J. Fitosociologia. Bases para el estudio de las comunidades vegetales. Madrid: H. Blume Ediciones, 1979. 820p.

COSTA, A. A.; ARAÚJO, G. M. Comparação da vegetação arbórea de cerradão e de cerrado na Reserva do Panga, Uberlândia, Minas Gerais. Acta Botanica Brasilica, v. 15, n. 1, p. 63-72, 2001.

COUTINHO, L. M. O conceito de cerrado.

Revista Brasileira de Botânica, v. 1, p. 17-23, 1978.

CRONQUIST, A. The evolution and classification of flowering plants. 2.ed. New York: The Botanical Garden, 1988. 555p.

FELFILI, J. M. et al. Análise comparativa da florística e fitossociologia da vegetação arbórea do cerrado sensu stricto na Chapada da Pratinha, DF - Brasil. Acta Botanica Brasilica, v. 6, n. 2, p. 27-46, 1993.

R. Árvore, Viçosa-MG, v.30, n.3, p.471-479, 2006
GOODLAND, R. Análise ecológica da vegetação do Cerrado. In: GOODLAND, R.; FERRI, M.G. (Eds.). Ecologia do Cerrado. Belo Horizonte: Itatiaia, 1999. p. 61-171.

HARIDASAN, M. Aluminium acumulation by some cerrado vegetation native species of Central Brasil. Plant and Soil, v. 65, p. 265-273, 1982.

HARIDASAN, M. Distribution and mineral nutrition of aluminium accumulating species in different plant communities of the cerrado region of central Brazil. In: SAN JOSÉ, R.R.; MONTES, R. (Eds.) La capacidad Bioprodutiva de Sabanas. Caracas: IVIC./CIET, 1988. p. 309-348.

LOPES, A. S. Solos sob cerrado:

características, propriedades, manejo. 2.ed. Piracicaba: Potafos, 1984. 162p.

MANTOVANI, W.; MARTINS, F. R. Florística do cerrado da Reserva Biológica de Moji Guaçu, SP. Acta Botanica Brasilica, v. 7, n. 1, p. 33-60, 1993.

MARTINS, F. R. Atributos de comunidades vegetais. Quid Teresina, v. 9, p. 12-17, 1990.

McCUNE, B.; MEFFORD, M. J. PC-ORD for windows: multivariate analysis of ecological data - verson 3.12. Gleneden Beach, Oregon: MJM Softmare Desiggn, 1997.

MENDONÇA, R. C. et al. Flora vascular do cerrado. In: SANO, S. M.; ALMEIDA, S. P. Cerrado ambiente e flora. Planaltina: EMBRAPA - CPAC, 1998. p. 289-556.

MÜELLER-DOMBOIS, D.; ELLENBERG, H. Aims and methods of vegetation ecology. New York: Willey \& Sons, 1974. 547p.

RATTER, J. A. et al. Observações adicionais sobre o cerradão de solos mesotróficos no Brasil Central. In: SIMPÓSIO SOBRE O CERRADO, 4., 1997, São Paulo. Anais... São Paulo: Universidade de São Paulo, 1977. p. 303-316.

RATTER, J. A. et al. Observations on the forests of some mesotrophic soils in central Brazil. Revista Brasileria de Botânica, v. 1, p. 47-58, 1978. 
RATTER, J. A.; DARGIE, T. C. D. Analysis of the floristic composition of 26 cerrado areas in Brazil. Edinburg Journal of Botany, v. 49, n. 2, p. 235-250, 1992.

RATTER, J. A. et al. Analysis of the floristic composition of the Brazilian Cerrado vegetation II: comparison of the woody vegetation of 98 areas. Edinburg Journal of Botany, v. 53, n. 2, p. 153-180, 1996.

RIBEIRO, J. F.; SILVA, J. C. S.; BATMANIAN, G. J. Fitossociologia de tipos fisionômicos de cerrado em Planaltina - DF. Revista

Brasileira de Botânica, v. 8, p. 131-142, 1985.

RIZZINI, C. T. Sobre as principais unidades de dispersão do cerrado. In: FERRI, M. G. (Coord.). SIMPÓSIO SOBRE O

CERRADO, 3,. São Paulo, Edgard Blücher, 1971.p. 117-132.

SHEPHERD, G. J. Fitopac 1: manual do usuário. Campinas: UNICAMP, 1996. não paginado.

SNEATH, P. H.; SOKAL, R. R. Numerical taxonomy. San Francisco: W. H. Freeman and Company, 1973.573p.

SILVA, L. O. et al. Levanatamento florístico e fitossociológico em duas áreas de cerrado sensu stricto no Parque Estadual da Serra de Caldas Novas, Goiás. Acta Botanica Brasilica, v. 16, n. 1, p. 43-53, 2002.
SILVA JÚNIOR, M. C. Composição florística, estrutura e parâmetros fitossociológicos do cerrado e sua relação com solo na Estação Florestal de Experimentação de Paraopeba, MG.1984. 130f. Dissertação (Mestrado em Ciência Florestal) - Universidade Federal de Viçosa, Viçosa, 1984.

SILVA JÚNIOR, M. C.; SILVA, A. F. Distribuição dos troncos das árvores mais importantes do cerrado na Estação Florestal de Experimentação de Paraopeba (EFLEX) - MG. Acta Botanica Brasilica, v. 2, n. 1-2, p. 107-126, 1988.

TER BRAAK, C. J. F. CANOCO - a FORTRAN program of canonical community ordination by (partial) (detrended) (canonical) correspondence analysis, principal componentes analysis and redundancy analysis (version 2.1). Wageningen: TNO - Institute of Applied Compter Science, 1988, 95p.

THIBAU, C. E. et al. Inventário preliminar expedido na Estação Florestal de Experimentação de Paraopeba em Minas Gerais. Brasil Florestal, v. 6, p. 34-71, 1975.

TOMÉ JÚNIOR, J. B. Manual para interpretação de análise de solo. Guaíba: Agropecuária, 1997.247p.

WEISER, V. L.; GODOY, S. A. P. Florística em um hectare de cerrado stricto sensu na ARIE - Cerrado Péde-Gigante, Santa Rita do Passa Quatro, SP. Acta Botanica Brasilica, v. 15, n. 2, p. 201-212, 2001. 